\title{
Orthographic Stereo Correlator on the Terrain Model for Apollo Metric Images
}

\author{
Taemin $\mathrm{Kim}^{1}$, Kyle Husmann ${ }^{1}$, Zachary Moratto ${ }^{1}$ and Ara V. Nefian ${ }^{1,2}$ \\ 1. NASA Ames Research Center, Moffett Field, CA, 94035 \\ 2. Carnegie Mellon University
}

\begin{abstract}
A stereo correlation method on the object domain is proposed to generate the accurate and dense Digital Elevation Models (DEMs) from lunar orbital imagery. The NASA Ames Intelligent Robotics Group (IRG) aims to produce high-quality terrain reconstructions of the Moon from Apollo Metric Camera (AMC) data. In particular, IRG makes use of a stereo vision process, the Ames Stereo Pipeline (ASP), to automatically generate DEMs from consecutive AMC image pairs. Given camera parameters of an image pair from bundle adjustment in ASP, a correlation window is defined on the terrain with the predefined surface normal of a post rather than image domain. The squared error of back-projected images on the local terrain is minimized with respect to the post elevation. This single dimensional optimization is solved efficiently and improves the accuracy of the elevation estimate.
\end{abstract}

\section{Introduction}

Topographical maps are an essential tool for scientists interested in exploring and learning more about planetary bodies like the moon or mars. These maps allow scientists do everything from identifying geological phenomena to identifying potential landing sites for probes or spacecraft.

Satellites and other spacecraft that visit planetary bodies of interest are usually equipped with a variety of sensors, some of which can be used to recover the topography of the planetary surface. LiDAR (Light Detection And Ranging) sensors give sparse (but highly accurate) measurements at periodic points called "posts." Raw images that are captured as the satellite orbits the planetary body can also be processed to create highly detailed topographical maps. By registering and aligning these two data sets, maps can be created that are both dense and accurate.

Given two images of the same scene taken from slightly different perspectives, the relative shift of objects from frame to frame (known as "disparity”) is related to distance of the object: far objects appear to move less than close objects. This phenomenon should be familiar, since the human brain uses this relationship to create depth perception from the differences in perspective seen by both eyes. Similarly, depth information from orbital imagery can be recovered in areas where the images overlap by matching points in the images that correspond to the same 3D location and measuring their disparity. By using the 


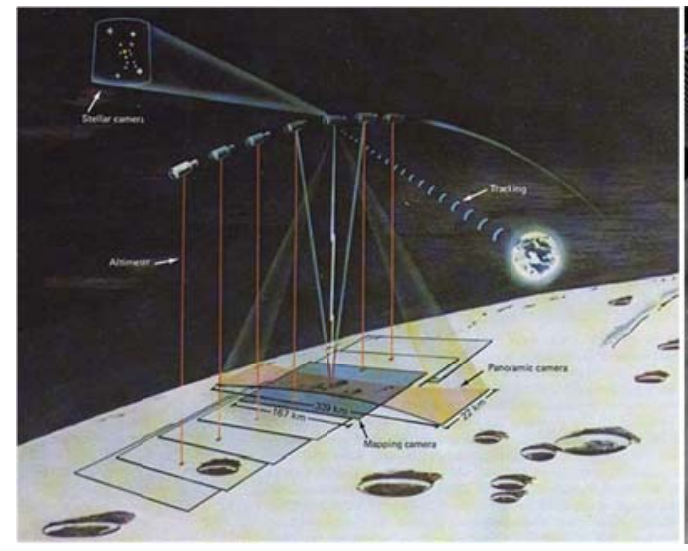

(a) The Mapping Cameras System

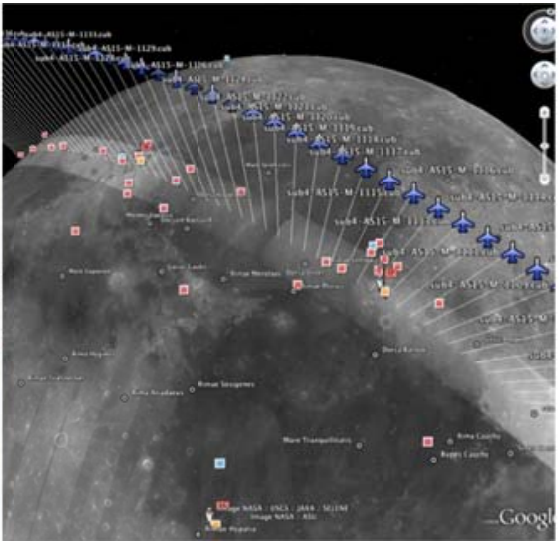

(b) Apollo 15 Orbit 33

Figure 1: AMC Data System. (a) The AMC captures a series of pictures of the Moon's surface. (b) Satellite station positions for Apollo Orbit 33 visualized in Google Moon.

disparity along with the location and orientation of the spacecraft as well as a mathematical model for the lens of the camera, the precise location of the 3D point can be calculated. This technique of using matching points between images to calculate 3D locations is known as "stereophotogrammetry" [6].

Before computers automated this task, points in orbital images were manually matched using mechanical stereoplotters. Now, computers can perform this once long and arduous process with minimal human interaction. For example, the Ames Stereo Pipeline [2, 5] is a collection of cartographic and stereogrammetric tools for automatically producing digital elevation maps (DEMs) from orbital images acquired with the Apollo Metric Camera (AMC) during Apollo 15-17 (Figure 1).

A new stereo correlation method is proposed on the terrain model to improve the accuracy of the DEM. This paper will address this problem by finding robust elevation from an image pair that minimizes the discrepancy between two back-projected patches on the local planar terrain. The proposed method determines the elevation by minimizing the squared error of the back-projected image patches. The accuracy of DEMs produced by IRG will thus be improved. The reconstructed DEMs from lunar orbital imagery are presented.

\section{Ames Stereo Pipeline}




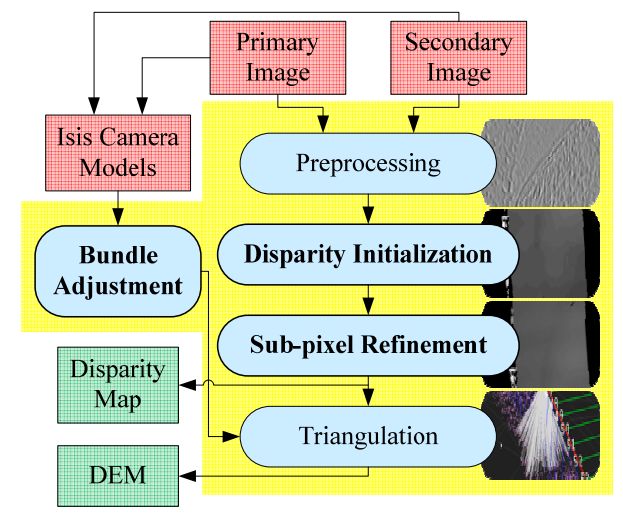

Figure 2: Dataflow of the Ames Stereo Pipeline. Preprocessing includes the registration and filtering of the image pair. A stereo correlator (disparity initialization and sub-pixel refinement) constructs the disparity map based on normalized cross correlation. DEMs are generated by a triangulation method in which corrected camera poses are used by bundle adjustment.

The Ames Stereo Pipeline (ASP) is the stereogrammetric platform that was designed to process stereo imagery captured by NASA spacecraft and produce cartographic products since the majority of the AMC images have stereo companions [10][18]. The entire stereo correlation process, from an image pair to DEM, can be viewed as a multistage pipeline (Figure 2). At the first step, preprocessing includes the registration to align image pairs and filtering to enhance the images for better matching. Triangulation is used at the last step to generate a DEM from the correspondences.

\subsection{Bundle Adjustment}

Before stereo correlation is performed, Bundle Adjustment (BA) corrects the three-dimensional postures of cameras and the locations of the objects simultaneously to minimize the error between the estimated location of the objects and their actual location in the images. Camera position and orientation errors affect the accuracy of DEMs produced by the ASP. If they are not corrected, these uncertainties will result in systematic errors in the overall position and slope of the DEMs. BA ensures that observations in multiple different images of a single ground feature are self-consistent.

In BA the position and orientation of each camera station are determined jointly with the 3D position of a set of image tie-points points chosen in the overlapping regions between images. Tie-points are automatically extracted using the SURF robust feature extraction algorithm [14]. Outliers are rejected using the random sample consensus (RANSAC) method [15]. The BA in ASP determines the best camera parameters that minimize the reprojection error [16]. The Levenberg-Marquardt algorithm is used to optimize the cost funciton [17]. 


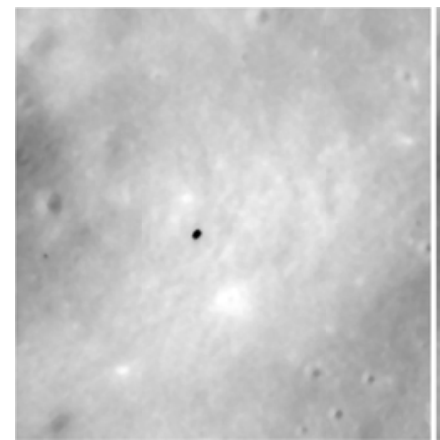

(a) Left Image

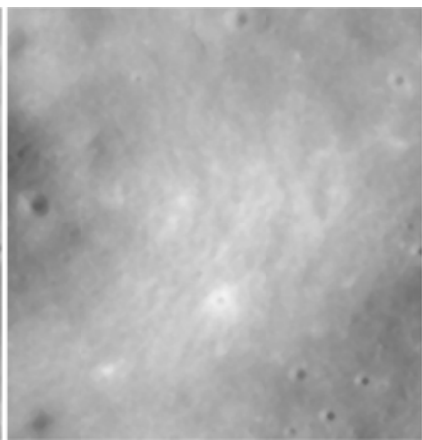

(b) Right Image

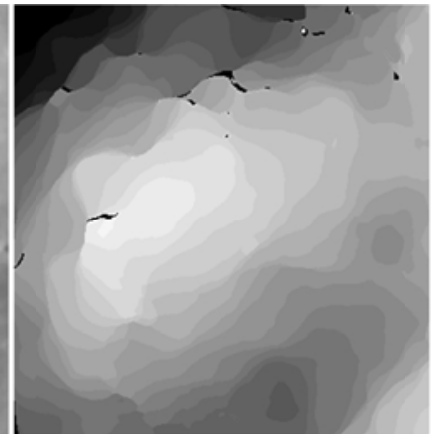

(c) Initialized Disparity Map

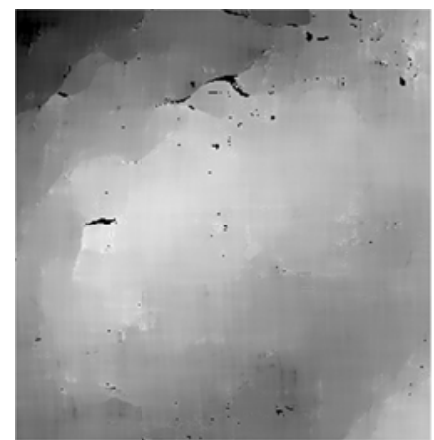

(d) Parabola Refined Map

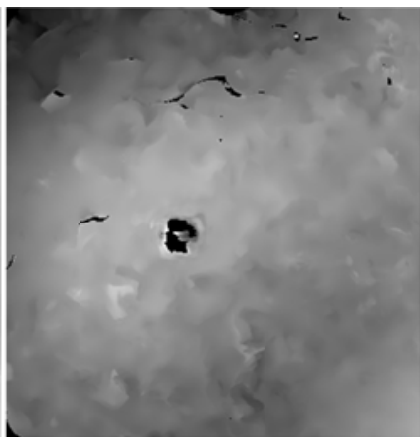

(e) Cauchy Refined Map

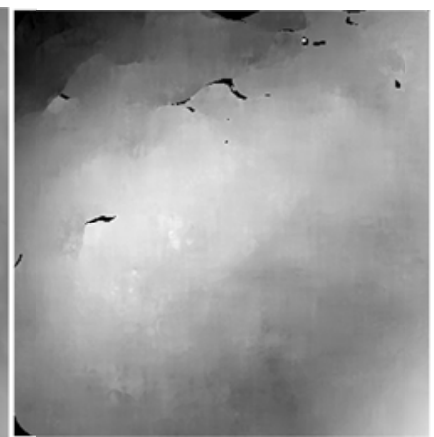

(f) Bayesian Refined Map

Figure 3: Stereo Correlation. (a-b) An image pair. (c-f) Horizontal disparity maps. (c) The fast discrete correlator constructs the coarse disparity map. (d-f) Refined disparity maps from the initialized map. (f) Bayesian sub-pixel correlator generates a smoother map than the others.

\subsection{Stereo Correlation}

Stereo correlation, which is the process at the heart of ASP, computes pixel correspondences of the image pair (Figure 3). The map of these correspondences is called a disparity map. The best match is determined by applying a cost function that compares the two windows in the image pair. The normalized cross correlation is robust to slight lighting and contrast variation in between a pair of images [11]. For large images, this is computationally very expensive, so the correlation process is split into two stages. (1) The disparity map initialization step computes coarse correspondences using a multi-scale 


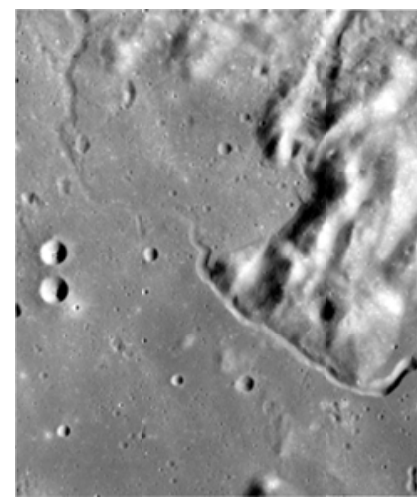

(a) Left Image

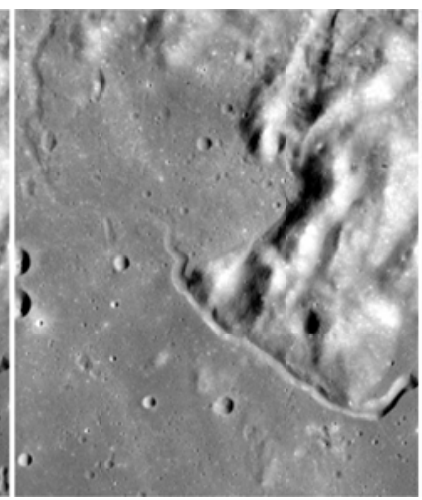

(b) Right Image

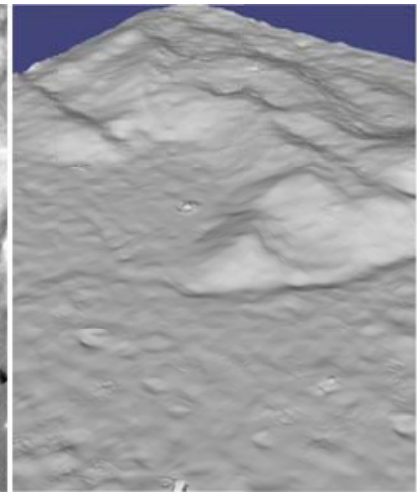

(c) Rendered DEM

Figure 4: Generation of DEM. (a) and (b) Apollo Metric Camera image pair of Apollo 15 site. (c) A DEM of Hadley Rille is rendered from an image pair.

search that is highly optimized for speed (Figure 3c). (2) Correlation itself is carried out by sliding a small, square template window from the left image over the specified search region of the right image (Figure 3d-f).

Refining the initialized disparity map to sub-pixel accuracy is crucial and necessary for processing real-world data sets [13]. The Bayesian expectation maximization (EM) weighted affine adaptive window correlator was developed to produce high quality stereo matches that exhibit a high degree of immunity to image noise (Figure 3f). The Bayesian EM sub-pixel correlator also features a deformable template window that can be rotated, scaled, and translated as it zeros in on the correct match. This affine-adaptive window is essential for computing accurate matches on crater or canyon walls, and on other areas with significant perspective distortion due to foreshortening. A Bayesian model that treats the parameters as random variables was developed in an EM framework [21]. This statistical model includes a Gaussian mixture component to model image noise that is the basis for the robustness of the algorithm. The resulting DEM is obtained by triangulation method (Figure 4).

\section{Orthographic Stereo Correlation}

To improve the DEMs produced by IRG, we proposed to define error measure in object domain rather than image domain for lunar orbital images. We propose to use a planar approximation of lunar terrain, which define the relationship of two views by a homography 


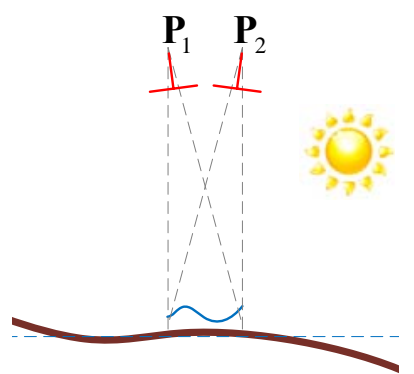

(a) Smooth Terrain

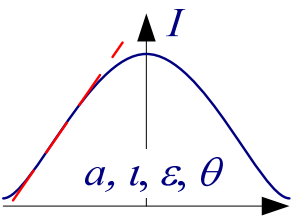

(c) Lunar reflectance

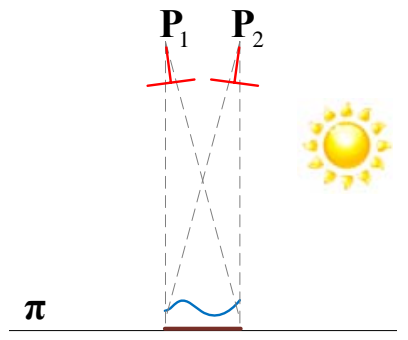

(b) Planar Terrain

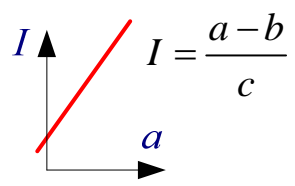

(d) Linear reflectance

Figure 5: Linear Approximation of Lunar Terrain and Reflectance. (a) Smooth local terrain in a small field of view is approximated by (b) planar one. (c) The lunar reflectance model is approximated by a linear function. (d) The linear reflectance is a linear function of surface albedo $(A)$.

representation. To model lunar reflectance, we propose to use a linear reflectance approximation. The statistical behavior of the photons is model by the normal distribution to derive the cost function that compares the two view windows. The proposed method will replace the pair-wise sub-pixel refinement and triangulation currently used in ASP.

A linear approximation of the lunar terrain and reflectance simplifies the correlation function. The lunar surface is smooth, but not flat (Figure 5a) and has its own reflectance (Figure 5c). The terrain is assumed to be locally planar because the correlation patches are determined to be small enough to cover the planar region (Figure 5b). Similarly, the lunar reflectance is assumed to be locally linear because geometric and photometric changes are small enough to form a linear relationship (Figure 5d).

A planar approximation of the terrain provides a homography representation of two-view correspondences. Suppose we have a textured plane that approximates the local terrain (Figure 7). Let $\pi$ be the surface plane with the normal vector $\mathbf{n}$ and distance $d$ from the origin, i.e., $\pi: \mathbf{n}^{T} \mathbf{x}=d$. Let $I$ be the orbital image and $f$ be the orthographic image of $I$ onto $\pi$. The surface albedo having linear relationship with $I$, i.e., $f(\mathbf{z})=b+c I(\mathbf{z})$ 
Figure 7: Homography Representation of Stere Correspondences. Homographies of the image planes induced by the local plane $\pi$, $\mathbf{H}_{i}: f_{i} \mapsto I_{i} \quad$ for $\quad i=1,2$, determine correspondences of the image pair.

for all $\mathbf{z} \in \pi$. Suppose we have an image pair viewing the same scene on $\pi$ with projection matrices $\mathbf{P}_{1}$ and $\mathbf{P}_{2}$.

To determine the geometric and reflectance parameters, we propose to minimize the squared error of all corresponding patches on the terrain. From the fact that the quantity of incident photons from the scene follows the normal distribution, the negative log-likelihood of scene radiance is derived:

$$
L(\mathbf{b}, \mathbf{c}, d ; \boldsymbol{\tau})=\int g(\mathbf{z}-\boldsymbol{\tau})\left\{f_{1}(\mathbf{z})-f_{2}(\mathbf{z})\right\}^{2} d \mathbf{z} .
$$

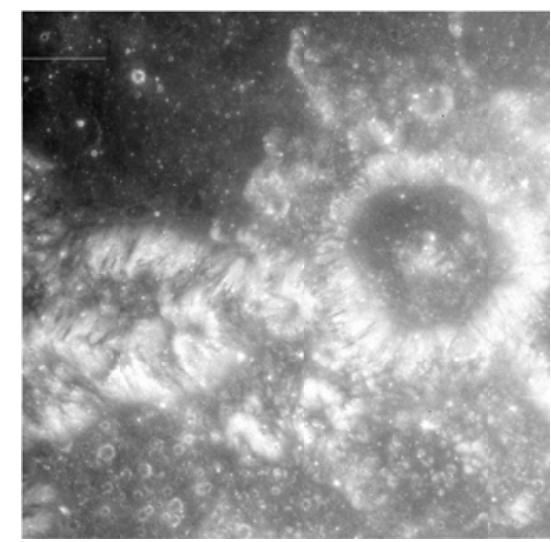

(a) AS15-M-1090

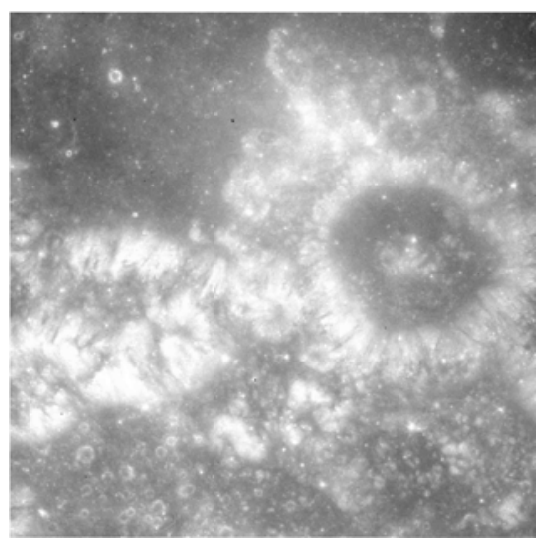

(b) AS15-M-1091

Figure 6: A Stereo Image Pair. Two images from Apollo 15 Orbit 33 imagery have different illumination and noises such as dust and missing data. 
where $f_{i}(\mathbf{z})=b_{i}+c_{i} I_{i}(\mathbf{z}), \mathbf{b}$ and $\mathbf{c}$ are coefficient vectors of linear reflectance, and $\langle\bullet\rangle_{\tau}=g(\tau)^{* \bullet}$ is the local Gaussian convolution operator. The optimizer of (1) may not exist in a closed form, but some parameters are simply optimized from the other parameters. To minimize the squared error, we propose to employ an alternating optimization scheme. First, we solve for $\mathbf{b}$ and $\mathbf{c}$ because there is a closed-form solution given $d$ in (1). Then we solve for $d$ in (1), while keeping surface albedo constant with the solutions from (1).The initialized disparity maps from ASP can be used to calculate the initial value of $d$.

\section{Experimental Results}

The orthographic stereo correlator is implemented based on the NASA Vision Workbench (VW). The NASA VW is a general purpose image processing and computer vision library developed by the IRG at the NASA Ames Research Center. The linear reflectance coefficients are estimated in the nested optimization process. Since this is a single dimensional optimization with respect to the elevation, we adopted a hybrid golden section and parabolic interpolation method. Figure 6 shows an image pair from Apollo metric images. A Gaussian window with scaling factor 5 is used for correlation window by $33 * 33$ pixels. Back-projected images are generated from an image pair with bicubic interpolation. The surface normal is determined by the radial direction of the local terrain because the lunar terrain is smooth enough.

Figure 8 a shows a stereo DEM constructed from integer disparity map. We can observe the pixel locking effect in the initial DEM with many holes. With this initial DEM, a refined DEM is reconstructed in Figure 8b. As you can see in the figure, the refined DEM gets rid of pixel locking artifact completely. There are small bumps and holes because the initial DEM has the large error and optimizer falls in local minima. Even though the surface normal is assumed roughly to have the radial direction of each post, the reconstructed terrain is reconstructed smooth enough and robust.

\section{Conclusion}

An orthographic stereo correlation method was proposed to reconstruct the accurate and dense Digital Elevation Models (DEMs) from lunar orbital imagery. The proposed method addresses this problem by making use of linearity in geometry and photometry to improve both the accuracy and robustness of the stereo correlation process. Given camera parameters of an image pair and an initial DEM, the DEM is refined to acquire the sub-pixel accuracy. A correlation window on the terrain with the predefined surface normal of a post is used to define the squared error of back-projected images on the local terrain. The squared error is minimized with respect to the post elevation. This forms a single dimensional optimization by nesting the linear reflectance optimization. For the future work, the surface normal can be estimated together with the elevation. 


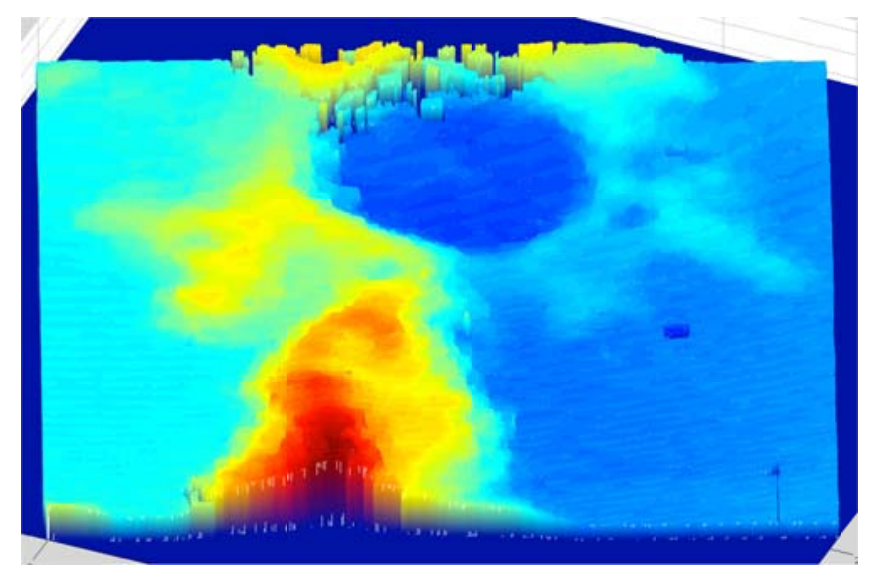

(a) Stereo Initial DEM

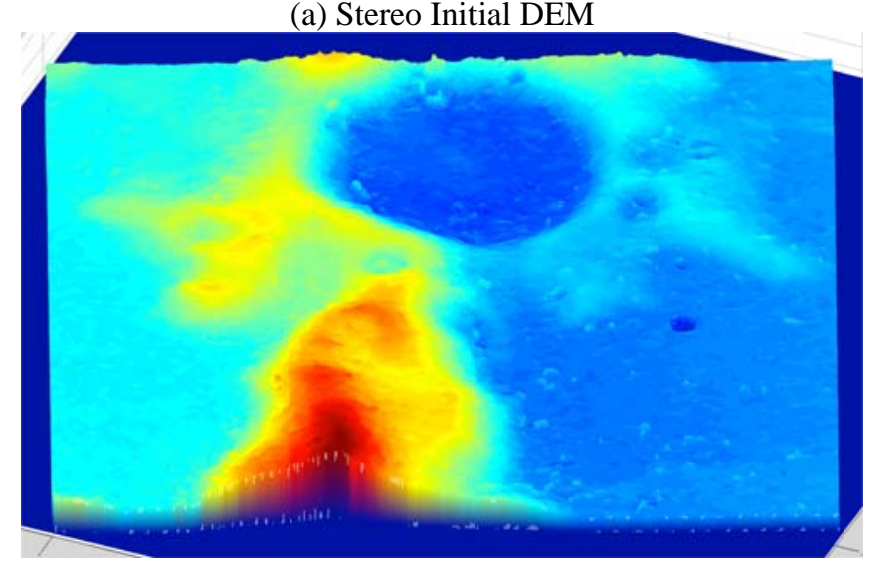

(a) Refined Dense DEM

Figure 8: Dense DEM Reconstruction. Color-mapped, hill-shaded DEM was reconstructed from from an image pair in Figure 6.

\section{References}

1. Noble, S.K., et al., The Lunar Mapping and Modeling Project. LPI Contributions, 2009. 1515: pp. 48. 
2. Mordohai, P. and G. Medioni. Dense multiple view stereo with general camera placement using tensor voting. in IEEE 3DPVT. 2004: pp. 725-732.

3. Thompson, C.M., Robust photo-topography by fusing shape-from-shading and stereo. 1992, Massachusetts Institute of Technology.

4. Price, K., Annotated computer vision bibliography. 1995: Institute for Robotic and Intelligent System School of Engineering (IRIS), University of Southern California.

5. Heipke, C. and C. Piechullek. Toward surface reconstruction using multi-image shape from shading. in ISPRS. 1994: pp. 361-369.

6. Cryer, J.E., P.S. Tsai, and M. Shah, Integration of shape from shading and stereo. Pattern recognition, 1995. 28(7): pp. 1033-1043.

7. Hapke, B., Bidirectional reflectance spectroscopy, 1, Theory. J. Geophys. Res, 1981. 86: pp. 3039-3054.

8. Minnaert, M., Photometry of the Moon. Planets and Satellites, 1961. pp. 213.

9. Buratti, B. and J. Veverka, Voyager photometry of Rhea, Dione, Tethys, Enceladus and Mimas. Icarus, 1984. 58(2): pp. 254-264.

10. Broxton, M.J., et al., The Ames Stereo Pipeline: NASA's Open Source Automated Stereogrammetry Software. 2009, NASA Ames Research Center.

11. Menard, C., Robust Stereo and Adaptive Matching in Correlation Scale-Space. 1997, Institute of Automation, Vienna Institute of Technology.

12. Sun, C., Fast stereo matching using rectangular subregioning and 3D maximum-surface techniques. International Journal of Computer Vision, 2002. 47(1): pp. 99-117.

13. Nefian, A., et al. A Bayesian Formulation for Subpixel Refinement in Stereo Orbital Imagery. in International Conference on Image Processing. 2009. Cairo, Egypt.

14. Bay, H., et al., Speeded-up robust features (SURF). Computer Vision and Image Understanding, 2008. 110(3): pp. 346-359.

15. Fischler, M.A. and R.C. Bolles, Random sample consensus: A paradigm for model fitting with applications to image analysis and automated cartography. Commun. Assoc. Comp. Mach,, 1981. 24(6): pp. 381-395.

16. Triggs, B., et al., Bundle adjustment - a modern synthesis. Lecture Notes in Computer Science, 1999. pp. 298-372.

17. Hartley, R. and A. Zisserman, Multiple view geometry in computer vision. 2003: Cambridge University Press.

18. Taemin Kim, Zachary Moratto and Ara V. Nefian, Robust Mosaicking of Stereo Digital Elevation Models from the Ames Stereo Pipeline,

19. F. Pedersini, P. Pigazzini, A. Sarti, S. Tubaro: 3D Area Matching with Arbitrary Multiview Geometry. EURASIP Signal Processing: Image Communication - Special Issue on 3D Video Technology", Elsevier, Vol. 14, No 1-2, October 1998, pp.71-94

20. M. J. Broxton, A. V. Nefian, Z. Moratto, T. Kim, M. Lundy, and A. V. Segal. 3D Lunar Terrain Reconstruction from Apollo Images. In Proceedings of the 5th ISVC 2009

21. A. Nefian, K. Husmann, M. Broxton, V. To, M. Lundy, M. Hancher. A Bayesian Formulation for Sub-pixel Refinement in Stereo Orbital Imagery. in Proceedings of the 2009 IEEE ICIP 2009 